\title{
La vigencia del modelo de propaganda de Herman y Chomsky en las protestas en Ecuador de octubre de 2019
}

The relevance of Herman and Chomsky's propaganda model during october 2019 protests in Ecuador

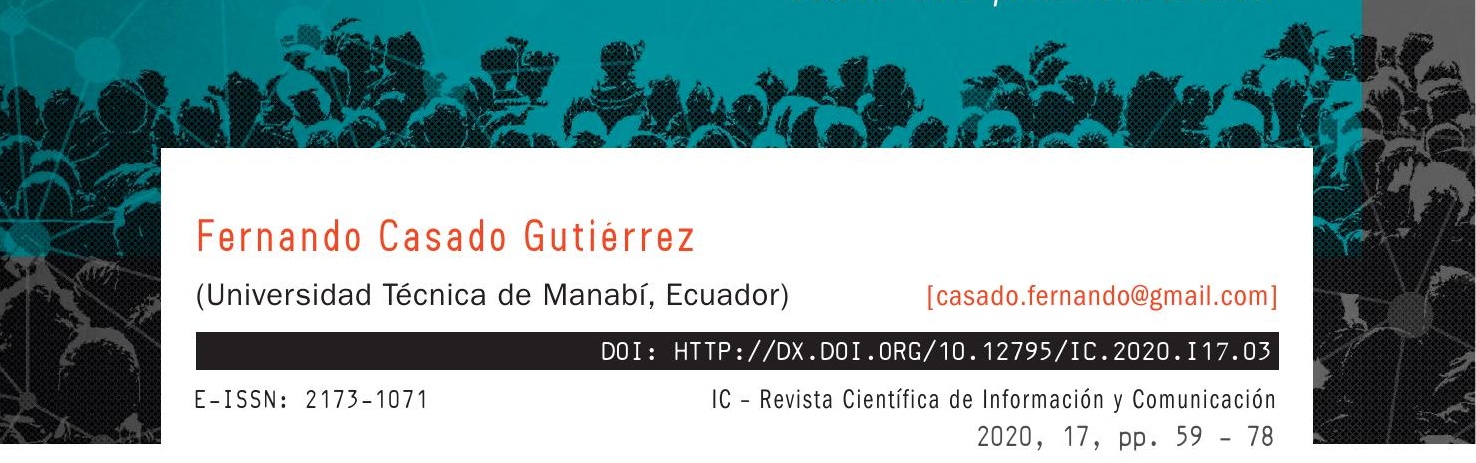

\section{Resumen}

El Modelo de Propaganda (MP) diseñado por Herman y Chomsky en 1988, en su obra los Guardianes de la Libertad, sigue contando con detractores y seguidores. Este modelo centrado en el comportamiento y actuación de los medios (Herman, 2000), critica frontalmente la ideología liberal y los intereses de las élites, lo que le situó en la periferia de los debates académicos. No obstante, a partir del nuevo siglo, algunos autores han profundizado a nivel teórico y práctico el MP (PedroCarañana, Boudry, Klaehn). Nuestra investigación pretende demostrar la actualidad del modelo a través del análisis (cuantitativo y cualitativo) de la cobertura y posicionamiento de los principales medios ecuatorianos de las protestas contra los planes de ajuste económico impuestos por el llamado decreto 883 que en octubre del año 2019 liberó el precio de los combustibles en Ecuador.

\section{Abstract}

The Propaganda Model (MP) designed by Herman and Chomsky in 1988, in their work the Guardians of Liberty, continues to have detractors and followers. This model centered on the behavior and performance of the media (Herman, 2000), frontally criticizes the liberal ideology and the interests of the elites, which placed it on the periphery of academic debates. However, since the new century, some authors have deepened the MP at a theoretical and practical level (Pedro-Carañana, Boudry, Klaehn). Our research try to demonstrate the relevance of the model through the analysis (quantitative and qualitative) of the coverage and positioning of the main Ecuadorian media of the protests against 
the economic adjustment plans imposed by the so-called Decree 883 that in October 2019 fuel price in Ecuador.

\section{Palabras clave}

Ecuador, Modelo de Propaganda, Chomsky \& Herman, Decreto 883, protestas indígenas.

\section{Keywords}

Ecuador, Propaganda Model, Chomsky \& Herman, legal Decree 883, natives protests.

\section{Sumario}

1. Introducción

2. El contexto en el que se desarrollaron los hechos.

3. Metodología

4. Análisis de los resultados

5. Aplicación del MP en Ecuador durante las protestas.

6. Conclusiones

7. Rererencias

8. Bibliografía

\section{Summary}

1. Introduction

2. The context in which the events took place.

3. Methodology

4. Analysis of the results

5. Application of the MP in Ecuador during the protests.

6. Conclusions

7. References

8. Bibliography 


\section{Introducción}

En 1988 los autores Chomsky y Herman escribieron su obra Manufacturating Consent, que en español fue traducida como Los guardianes de la libertad, en la que construyeron lo que Ilamaron el Modelo de Propaganda (MP) cuyo principal postulado es que los medios de comunicación por sus características estructurales aplican cinco filtros que orientan los hechos que serán publicados y la forma en que se publicarán.

Estos cinco filtros serán: 1) la envergadura y propiedad de los medios; 2) la publicidad como fuente principal de ingresos de los medios; 3 ) la dependencia de los medios de ciertas fuentes de información; 4) los "correctivos" para disciplinar a los medios; 5) el anticomunismo, que fue modificado posteriormente en la revisión del modelo en el año 2002 por "la ideología de libre mercado" (Klaehn, 2018) y como "convergencia en la ideología dominante" (Pedro, 2008) que es el término utilizado en el presente trabajo. La aplicación de estos filtros tendrán como resultado la "el dominio de los medios de comunicación por parte de la élite y la marginación de la disidencia" (Chomsky \& Herman, 1988, p. 22). El quid de la aplicación de estos filtros se traducirá en la existencia de víctimas "dignas" e "indignas", que serán las que acaparen la atención de los medios de comunicación de élite de forma dicotómica dependiendo de sus intereses y presiones (Kennis, 2009, p. 387).

A la hora de aplicar el MP, tal y como aclaró el propio Herman en un estudio retrospectivo de su trabajo, se debe tener en cuenta que se analiza y estudia "el comportamiento y actuación de los medios de comunicación, pero no los efectos de los medios" (2000). El MP se expone como "un sistema de mercado dirigido", dado que existen tan pocos actores entre los grandes medios que en un momento determinado pueden actuar al unísono. Para los creadores del MP los propietarios de los medios en la mayoría de los casos "hacen cosas parecidas porque ven el mundo desde la misma perspectiva" y están sometidos a los mismos límites e incentivos, lo que resulta en que las historias sean narradas u ocultadas de manera similar y por los mismos motivos (Chomsky \& Herman, 1988, p. 14). El MP argumenta que el resultado del comportamiento de los medios será el de autocensura sin que se produzca coerción (Klaehn, 2003). Lo que conecta el MP con la reducción de la libertad humana y la escuela crítica con autores como Horkheimer y Adorno (Pineda, 2001).

EI MP no tuvo una recepción entusiasta en el mundo académico tras su publicación y fue en la mayoría de los casos ignorado, pero también criticado. Para muchos autores las críticas tendrían su origen en la perspectiva anti-élite del MP (Pedro, 2009). Otros consideran que, aunque el MP es válido en términos generales, requiere ser adaptado 
a "la naturaleza de las presiones y contingencias de la producción de noticias que surge de las realidad política y económica de la democracia capitalista" (Sparks, 2007).

Entre las críticas podemos destacar que el MP es acusado de ser útil cuando es aplicado en Estados Unidos y con relación a la cobertura de los asuntos internacionales, pero no tendría validez para asuntos domésticos y otras realidades como Europa y países con sistemas mediáticos y estructuras políticas distintas a la estadounidense (Corner, 2003). No obstante, estos argumentos han sido rebatidos por Klaehn, para quien existen suficientes evidencias empíricas que demostrarían la aplicabilidad del MP en distintas realidades (2003). En el contexto español y el surgimiento de Podemos, Labio-Bernal afirma que el MP "continúa siendo perfectamente válido para el análisis de la ideología de los medios y su aplicación traspasa los contextos fuera de Estados Unidos" (2018); mientras que Bergman realizó un estudio aplicando el modelo a los medios holandeses (2014).

Se ha acusado al MP de ser poco preciso en la caracterización de los filtros, que privilegian los factores estructurales, pero evitan y marginan la intencionalidad y los micro-procesos que se dan en las salas de redacción (Boyd-Barrett, 2004). En ocasiones se ha señalado que el MP podría llegar a confundirse con el Modelo de los Gatekeepers, pero a diferencia de este, el MP no asume que quienes "trabajan en los medios de comunicación de manera rutinaria toman decisiones conscientes para alinearse con los intereses de las élites" (Klaehn, 2003). En ocasiones también se ha acusado de ser un modelo "conspiratorio" (Robinson, 2018) y simplista, ya que las noticias "son un producto negociado, una construcción social, sobre las que ni el gobierno ni nadie tiene un control total" (Lang \& Lang, 2004). Otras observaciones han identificado paradojas en la contradicción entre las descripciones lingüísticas de la tradición de "Chomskiana" y el análisis del discurso de las noticias en el MP (Lukin, 2013). Autores como ÁlvarezPeralta analizando el caso de España, han encontrado limitaciones en la aplicación del MP en ciertas dinámicas de comunicación, como las redes sociales, particulares culturas políticas, como la española y contextos de crisis e inestabilidad (2018).

Independientemente de las críticas que pudieran ser realizadas al MP y que han generado hasta el momento intensos debates, coincidimos con autoras como Elli Lester que consideran que el modelo es útil para explicar por qué lo medios de comunicación “no fomentan la participación política de los ciudadanos, y además predice que tipo de cobertura responde a situaciones específicas" (1992).

Muchas de las críticas han dado lugar a que sus autores hayan tratado de perfeccionar el MP proponiendo nuevos filtros. Joan Pedro establece como un filtro autónomo el uso de la tecnología, que ha servido "como norma general (aunque con 
algunas desviaciones puntuales) a los intereses del poder" (2009). Boyd-Barrett considera que un sexto filtro debería ser la compra de los periodistas, lo que relaciona con su crítica a la falta de atención que dedica el MP a la intencionalidad (2004). Broudy \& Tanji, tras analizar las filtraciones de Manning, Snowden y Assange entre otros, proponen que se establezca como un filtro los sistemas de seguridad pues conceden "a la élite el monopolio virtual sobre la definición de las fronteras aceptables del debate público y control sobre la "correcta" interpretación de conceptos claves e ideas" (2018).

Pese a que inicialmente se le haya prestado poca atención al modelo, la llegada del nuevo siglo ha traído una fructífera cantidad de estudios que han utilizado como referencia el MP diseñado por Chomsky y Herman. Uno de los temas sobre el que se han realizado un mayor número de estudios aplicando el MP ha sido la guerra de Irak. En el contexto de la crítica al comportamiento de los medios estadounidenses Boyd-Barrett usó el MP en el marco de la invasión a Irak y la cobertura de la corresponsal del diario New York Times, Judith Miller (2004). La guerra en Irak analizada desde el MP constituyó para otros autores una afectación para la propia democracia por la forma en que ciertos temas han sido sustraídos del debate político (Taylor Jackson \& Ronald Stanfield, 2004). Pero no solo desde el ámbito de los medios estadounidenses, sino también desde los británicos ha sido aplicado el MP en relación con la guerra de Irak (Freedman, 2009).

El MP ha sido utilizado en estudios de muy variada índole: Labio-Bernal concentró su análisis en el quinto filtro del Anticomunismo y cómo fue aplicado por los medios de comunicación en España para denostar el partido político Podemos cuya aparición amenazaba el bipartidismo político español (2018); otros estudios se centraron en comprobar cómo los medios de comunicación han evitado abordar en sus historias el cambio climático (Good, 2008); pero también en la cobertura negativa de los atletas profesionales respecto a dueños de equipos deportivos (Pollick, 2018); la crisis financiera, recesión y austeridad en el Reino Unido en el año 2008 (Mullen, 2018); el golpe de estado en Venezuela en el año 2002 (Sierra, 2018); e incluso la industria cinematográfica de Hollywood; los mercados financieros, aunque en este caso solo algunos filtros fueron de utilidad (Thomson, 2009); o el uso de bombas atómicas (Rai, 2018).

Andrew Kennis realizó un estudio que tiene muchos puntos en común con nuestra investigación, al aplicar el MP a la cobertura por parte de la prensa estadounidense de las protestas indígenas en Ecuador contra el gobierno de Jamil Mahuad en el año 2000. El estudio de Kennis concluye que los pueblos indígenas recibieron un tratamiento de "víctimas indignas" por la prensa y que los acuerdos entre el gobierno y el FMI fueron silenciados en la cobertura (2009). 
Nuestro estudio pondrá de manifiesto que el MP todavía tiene vigencia y que puede ser aplicado a realidades contemporáneas y a temas de carácter doméstico. Para ilustrar nuestro trabajo tomaremos como referencia las protestas que se produjeron en Ecuador a principios de octubre del año 2019 como respuesta a las medidas de ajuste económico que significó la entrada en vigor del decreto 883.

\section{El contexto en que se desarrollaron los hechos}

El martes 1 de octubre del año 2019 el presidente de Ecuador, Lenín Moreno, en cadena nacional anunció una serie de medidas económicas, entre ellas la liberalización del precio de los combustibles que dejarían de estar subsidiados tras la inmediata entrada en vigor del decreto 883 (El Comercio, 2019).

Estas medidas se impusieron en un contexto de crisis económica y acuerdos para la obtención de crédito con el Fondo Monetario Internacional (FMI). Es preciso señalar que el presidente Lenín Moreno llegó a la Presidencia de Ecuador como un gobierno de continuidad de su antecesor, Rafael Correa, crítico del neoliberalismo, pero tras tomar el poder, dio un viraje a posiciones liberalizadoras de la economía basadas en un Estado débil.

La liberalización de los precios de los combustibles (diésel, ecopaís y extra), eliminó unos subsidios que habían estado vigentes por más de cuatro décadas y que según el gobierno ahorrarían 1.300 millones al Estado, de esta forma:

E E precio del galón de extra y ecopaís subió de 1,85 a 2,3 dólares.

E El precio del galón de diésel subió de 1,03 a 1,97 dólares, aunque en el mercado internacional el precio era de 2,11 dólares.

Entre las compensaciones diseñadas por el gobierno se anunció:

Alrededor de 300 mil familias recibirían 15 dólares mensuales a través de distintos bonos, en principio las más pobres del país.

E Eliminación o reducción de aranceles para la importación de maquinaria, equipos y materias primas agrícolas e industriales. 
Eliminación de aranceles a la importación de celulares, tabletas y computadores, que estaban gravados con el 15 y $10 \%$ de aranceles, que habría sido posteriormente la única medida que se ha llevado a cabo (Orozco, 2019).

Asignación de 1.000 millones de dólares para créditos hipotecarios desde noviembre, a una tasa de 4,99\%.

- Medidas laborales de flexibilización para el sector público:

- Contratos ocasionales se renuevan con $20 \%$ menos de remuneración.

- Reducción del número de días de vacaciones de 30 a 15 días.

Pese a todas las medidas anunciadas, el Decreto 883 únicamente liberó de manera inmediata los precios del combustible. Las medidas compensatorias para los más pobres que buscaron contrarrestar el golpe de la subida de los combustibles mediante bonos o la adquisición de vivienda nunca fueron concretadas en medidas ejecutivas. Es decir, el resto de las medidas siempre fueron una incógnita y durante el periodo de vigencia del Decreto 883 no se materializaron en ninguna propuesta concreta ni legislativa ni ejecutiva.

Dado el impacto inmediato y devastador para la economía de una gran cantidad de ecuatorianos que se vieron afectados duramente:

El 9,5\% de la población, es decir,1,6 millones de ecuatorianos, vive con un dólar al día, lo cual sitúa a estas personas en una situación de altísima vulnerabilidad y en situación de pobreza extrema (INEC, 2019).

— A ellas se añade el $25,5 \%$ de la población en situación de pobreza por ingresos, por lo tanto, en total, casi 5 millones de personas, viven con menos de un salario básico en Ecuador que se sitúa en torno a 400 dólares (INEC, 2019).

Para las personas en situación de pobreza, se incrementaron inmediatamente los costos de transporte y productos básicos, y para los pequeños productores campesinos, también se encarecieron substantivamente los costos de producción y transporte. El Decreto no tomó en consideración la focalización o impacto de la liberalización de los combustibles en las personas más humildes.

La respuesta fue una dura protesta social que inició el día después de anunciadas las medidas, el 2 de octubre, y que tuvo una duración de 11 días, hasta que 
el 12 de octubre el gobierno decide derogar el Decreto e iniciar un proceso de diálogo con los líderes de la protesta. El costo fue muy alto, según la Defensoría del Pueblo (2019):

Once personas fallecidas;

1192 personas detenidas;

1340 personas heridas, en distintos grados de gravedad;

2.500 millones dólares en daños un 3\%, según Luis Almagro, secretario de la OEA (El Comercio, Lenín Moreno pide estrategia regional para defender la democracia, 2019), pero solamente en material militar las pérdidas ascenderían a más de 9 millones de dólares (Sputnik, 2019).

Tras el primer día de protestas se decretó el estado de excepción que fue acompañado posteriormente por toques de queda parciales fuertemente criticados por la Defensoría del Pueblo (2019). La Corte Constitucional avaló por unanimidad la legalidad de la medida del estado de excepción, solo modificándola al reducir su tiempo de vigencia de 60 a 30 días. El estado de excepción no fue derogado pese a la eliminación del Decreto y la desactivación de la protesta (El Universo).

Durante los días que duró la protesta se redobló la persecución contra los líderes del grupo político de la Revolución Ciudadana conocidos como los correístas, por su afinidad con el expresidente Rafael Correa. No solo se realizaron numerosos ataques en cadenas nacionales del gobierno a este grupo político, sino que se produjeron tras el inicio de las negociaciones varias detenciones, como la prefecta de Pichincha Paola Pabón y dos de sus colaboradores. El miedo a que las detenciones se pudieran seguir produciendo provocó que tres asambleístas de la bancada correísta solicitaran refugio en la embajada de México en Ecuador (EFE, 2019).

El gobierno de Lenín Moreno presionado por las protestas decidió iniciar un proceso de diálogo, pero a posteriori, cuando durante los primeros meses de su gobierno había propugnado un diálogo con todos los sectores del país e incluso promovió una consulta popular en el año 2018, y aseguró entonces que sería repetida en momentos posteriores: "No será la única consulta, el pueblo será consultado siempre" (Sputnik, Presidente de Ecuador anuncia que realizará más consultas populares, 2017), lo que hasta el momento no ha ocurrido. 
La fuerte represión ocurrida durante los días de la protesta para imponer las medidas de ajuste económico del gobierno ecuatoriano, fueron duramente criticadas por diversas voces entre quienes se encontró uno de los padres del MP, Noam Chomsky (Chomsky, Majfud, Castells, \& Cafassi, 2019).

\section{Metodología}

Nuestra investigación pretende demostrar la vigencia del MP de Chomsky y Herman, a través de un estudio de carácter cuantitativo y cualitativo para ver si existe correspondencia en tres hipótesis planteadas:

1. La élite económica y política apoyó las medidas de ajuste económico a pesar del sufrimiento que pudiera causar al sector de la población más desfavorecidas.

2. Se produce una aplicación en Ecuador durante las protestas contra la imposición de ajuste económico de los filtros del MP.

3. Desde los medios de comunicación se establecieron víctimas dignas e indignas durante los días que duró el conflicto de acuerdo con la conveniencia de las narrativas de los medios de comunicación.

Un factor importante que debemos tener en cuenta al considerar MP es que los elementos históricos y los que tengan relación con la economía política toman importancia al realizar los análisis mediáticos, independientemente de la aproximación específica que decidamos al aplicar el modelo (Klaehn, 2009).

En la aplicación del MP y al verificar el cumplimiento de las hipótesis, comprobaremos si existe una correspondencia y apoyo entre lo expresado en las cadenas presidenciales durante los días que duraron las protestas y el posicionamiento tomado por algunos de los medios más importantes de Ecuador. Analizaremos cuatro medios de comunicación: dos de prensa y dos de televisión. Los diarios escogidos son los dos líderes de la prensa generalista en Ecuador: El Comercio y El Universo; y de televisión los dos canales privados con mayor difusión: Ecuavisa y Teleamazonas.

Nos importa saber cuál es la opinión que los medios trataron de imponer a sus audiencias y hasta qué punto existió una pluralidad o falta de ella en los enfoques durante las 
protestas. Tomaremos por tanto en consideración las columnas de opinión y editoriales de la prensa y los principales programas de opinión diarios en los canales de televisión: "Contacto Directo" en el caso del canal Ecuavisa; y "Los Desayunos de 24 Horas" de Teleamazonas.

El corpus de estudio fue de 16 programas de televisión (ocho por cada uno de los canales de televisión que fueron emitidos durante las protestas) y 151 piezas periodísticas publicadas en los dos diarios analizados. El periodo considerado fue, en el caso de los programas de televisión desde el día después de anunciadas a las medidas económicas, es decir el 3 de octubre, hasta el día siguiente que se estableció el diálogo entre el gobierno y Ios líderes indígenas. Para el caso de los programas de televisión hemos clasificado el perfil de los invitados a los programas, así como su apoyo o rechazo a las medidas económicas, además de la posición y editoriales expresados por los entrevistadores. En el caso de la prensa, el periodo de análisis lo empezamos un día después, el 4 de octubre debido a que fue a partir de este día que empiezan los diarios a hacerse eco de las medidas económicas tomadas y sus repercusiones. Para el análisis de los editoriales y artículos de opinión de la prensa se tomó en consideración los principales temas y encuadres acompañados de un análisis de contenido de los artículos.

\section{Análisis de los resultados}

En cuanto a los resultados de tipo cuantitativo arrojados por la prensa, del total de los 151 artículos de opinión y editoriales publicados en los 11 días seleccionados, un total de 114 tuvieron relación con el Decreto 883 y/o las consecuencias de su entrada en vigor, es decir el $75 \%$. Sin embargo, al dividir por géneros periodísticos observamos que el total de los 22 editoriales trataron el ajuste económico y sus consecuencias. Entre Ios artículos de opinión El Comercio publicó un total de 76 columnas, de las que un $80 \%$ estuvieron relacionados con el ajuste económico, mientras que, de las 64 columnas de El Universo, un 65\% tuvieron relación con este tema.

En términos cualitativos, de los editoriales el tema más abordado fue el vandalismo y la violencia que las protestas desencadenaron, que aparece en un total de catorce editoriales como el tema principal, y en segundo lugar el respaldo a las medidas económicas del gobierno. Además, en dos editoriales el vandalismo es atribuido al correísmo como tema principal, y en otros dos como tema secundario.

Entre los artículos de opinión, el respaldo a las medidas económicas como tema principal ocupó un $35 \%$ de las notas, un $28 \%$ se dedicó a la violencia y el vandalismo. 
Adicionalmente un $18 \%$ culpó del vandalismo, la violencia e incluso de promover un golpe de Estado, al expresidente Rafael Correa y los correístas.

En lo que a los programas de televisión se refiere, se pudo observar en primer lugar una clara línea editorial a favor de las medidas económicas que defendieron los entrevistadores en sus comentarios y aperturas de las entrevistas. De los entrevistados, alrededor de un tercio fueron personas que estuvieron en contra de las medidas, principalmente representantes de trabajadores y transportistas $y$, en menor medida, representantes indígenas. Los dos tercios restantes de los invitados estuvieron a favor de las medidas entre representantes del gobierno, representantes del sector empresarial, políticos de oposición y analistas. Algo más de la mitad de quienes estuvieron a favor de las medidas económicas fueron voceros del gobierno, es decir un tercio aproximadamente del total, quienes tuvieron un mayor espacio en estos programas de opinión. En concreto el secretario de la Presidencia, José Augusto Briones, llegó a tener tres apariciones en los dos canales de televisión analizados.

Por último, algo importante a destacar es que todos los comentaristas e invitados coincidieron en una animadversión contra el correísmo e hicieron comentarios en su contra, al mismo tiempo que ningún vocero de este movimiento político fue invitado a ninguno de los programas.

Desde el anuncio de las medidas el 2 de octubre, hasta el día después del inicio del diálogo, el 14 de octubre, se emitieron un total de siete cadenas presidenciales. Durante el periodo de protestas el presidente de la República en cuatro de ellas acusó al expresidente Rafael Correa y al mandatario venezolano, Nicolás Maduro, de estar detrás de Ios actos vandálicos y la violencia generada, pero sin presentar pruebas. Otros argumentos muy utilizados durante las cadenas fue asegurar que las medidas económicas tomadas iban dirigidas al beneficio de los más pobres, acabar con el contrabando de combustible y sus mafias y asegurar en todo momento que nunca daría marcha atrás en la liberalización de los precios de los combustibles, como finalmente hizo.

\section{Aplicación del MP en Ecuador durante las protestas}

En esta última parte de nuestro trabajo comprobaremos si se han cumplido las hipótesis más arriba planteadas. La primera de estas estableció que la élite económica y política apoyó las medidas de ajuste económico a pesar del sufrimiento que pudiera causar a las mayorías más desfavorecidas. La propuesta responde a los planteamientos de 
Chomsky \& Herman que afirman que el consenso de la élite se refleja en la conformidad de los medios de comunicación (Mullen, 2018).

Los medios de comunicación tomaron una postura de total connivencia con el poder político y económico difundiendo y afianzando sus narrativas en todo momento. Lo podemos observar, en primer lugar, en que las medidas de corte liberal se ajustan a los reclamos de la élite económica, y su presentación como necesarias y adecuadas, aunque dolorosas para muchos, fue la línea seguida por los medios de comunicación. Entre los voceros en los programas de televisión predominaron los que defendían los planes de ajuste económico y entre la opinión de editoriales, columnistas y entrevistadores también fue mayoritaria la argumentación a favor de las medidas. Las voces críticas, pese a las protestas generalizadas en Ecuador, fueron minoritarias entre los invitados a los programas de opinión y prácticamente inexistentes en la prensa.

La segunda hipótesis planteada tiene que ver con la aplicación de los filtros establecidos por Chomsky \& Herman en su MP que son:

1. La envergadura y propiedad de los medios

La estructura y altos niveles de concentración de los medios de comunicación ha sido ampliamente estudiado y constatado (Bagdikian, Reig, Serrano entre otros) y en este sentido Ecuador no iba ser una excepción.

Pese a los intentos por distribuir el espacio radioeléctrico en partes iguales entre el sector público, privado y comunitario tras la entrada en vigor de la Ley Orgánica de Comunicación de 2013 (ley que ya ha sido modificada) y a que la constitución vigente obligue la separación entre el capital financiero y los medios de comunicación, "la concentración de medios en el país andino se caracteriza por la propiedad en manos de unas pocas familias, y en mayor medida, de grupos económicos" (Casado \& Sánchez, 2017, pp. 27). Por lo tanto, el primer filtro es fácilmente identificable.

2. La dependencia de la publicidad

Este filtro está íntimamente relacionado con el anterior (Pedro, 2008), la tendencia de la concentración de las empresas publicistas se ha ido produciendo de forma similar a los medios de comunicación. Para el año 2019 la publicidad sigue constituyendo un mecanismo de influencia y presión de estas empresas sobre los medios de comunicación, aunque hoy día el internet acapare prácticamente la mitad de las inversiones totales según Zenith (Insider, 2019). La tendencia 
mundial es la disminución de la inversión en publicidad en los medios tradicionales, mientras aumenta la inversión en publicidad digital. Las empresas de telecomunicaciones serían las que lideran la inversión en publicidad en los medios digitales (Admetricks, 2018). Esta tendencia internacional también estaría afectando a Ecuador.

3. El suministro de noticias a los medios de comunicación por parte de las fuentes oficiales

Los autores Chomsky y Herman establecen como uno de los filtros del MP la relación simbiótica entre los medios de comunicación y las fuentes oficiales "tanto por la necesidad económica como por reciprocidad de intereses" (1988, p. 50). En la actual coyuntura observamos que existe en una excesiva visibilidad de las fuentes gubernamentales. En la coyuntura analizada podemos ver la existencia de este filtro en el hecho de que las fuentes oficiales ocuparon un gran espacio entre los voceros invitados a los espacios de opinión, un tercio, y además entrevistadores, editoriales y columnistas repitieron en gran medida las narrativas del gobierno. Existe por tanto una reproducción del mensaje de las fuentes oficiales.

4. El flack o las contramedidas para disciplinar a los medios (reforzadores de opinión)

Este filtro se refiere a las medidas tomadas por los gobiernos para disciplinar a los medios de comunicación. Tomando como referencia la teoría del garrote y la zanahoria, este filtro se correspondería con el garrote. Las medidas tomadas por el gobierno pueden adoptar distintas formas, desde llamadas telefónicas a visitas al medio, adoptando distintos niveles de "queja, amenaza y acción punitiva" (Chomsky \& Herman, 1988, p. 63).

Sería difícil poder evaluar si el gobierno ha llevado a cabo en la coyuntura actual acciones concretas para disciplinar a los medios de comunicación, pero sí hubo ciertas acciones contra medios que podrían considerarse disidentes de la línea oficial que se enmarcarían desde nuestro punto de vista en este filtro, aunque no forman parte de la muestra de piezas analizadas en la investigación. Mencionar entonces, el cierre de la señal de Radio Pichincha después de que el 8 de octubre sus instalaciones fueran allanadas por la policía por haberse cometido el presunto delito de incitación a la discordia entre ciudadanos (El Universo, "Fiscalía allana instalaciones de Radio de la Prefectura de Pichincha por 
incitación a la discordia entre ciudadanos”, 2019). La Radio Pichincha tiene gran cantidad de radioescuchas en la capital Quito, donde se produjeron el grueso de las manifestaciones, es pública y pertenece a la prefectura de la provincia, es decir depende de la prefecta Paola Pabón, con filiación política correísta, quien ganó las elecciones seccionales de marzo de 2019. Radio Pichincha siempre ha tenido una posición crítica hacia el gobierno por lo que ante la imposibilidad de controlar su línea editorial el gobierno pudo haber ordenado su allanamiento. Pocos días después, la prefecta Paola Pabón sería detenida "para investigaciones", (El Universo, "Paola Pabón, Prefecta de Pichincha, fue detenida para investigaciones", 2019) después de haber sido acusada directamente por el mandatario Lenín Moreno de estar involucrada en un golpe de estado en su contra junto al ex presidente Correa y el presidente Maduro (Ecuador Tv, 2019).

5. El anti-comunismo como mecanismo de control (hoy, convergencia en la ideología dominante)

El filtro final, la convergencia en la ideología dominante, en el sentido de que todo lo que sea percibido como una amenaza al sistema representativo democrático liberal y economía de libre mercado será duramente criticado en los medios de comunicación. Los medios de comunicación juegan el rol fundamental al reforzar el consenso de la élite económica y política.

La expresión de la convergencia la vemos claramente en cómo los medios de comunicación dieron un total espaldarazo a las medidas económicas y cómo sus encuadres hicieron hincapié, o bien en la violencia y el vandalismo, o bien en esa misma violencia, pero buscando el chivo expiatorio del correísmo. Al mismo tiempo, el vandalismo se achacó en medios de comunicación a los manifestantes y justificó u ocultó la acción de las fuerzas de seguridad del Estado. En consecuencia, los medios silenciaron aquellos aspectos que pudieran dejar en evidencia las falencias de las medidas o los perjudicados por estas, y se centraron en visibilizar la lucha contra el contrabando de combustible. Las voces disidentes y que podrían contradecir la convergencia de la ideología dominante tampoco tuvieron cabida en los medios de comunicación.

La tercera hipótesis tiene relación con el establecimiento de víctimas dignas de la atención de los medios comunicación, y víctimas indignas a las que se les negaría la representación o ésta sería deformada. 
Como víctima digna podemos mencionar la agresión del periodista Freddy Paredes quien fuera herido por una pedrada de los manifestantes y al que se le dedicó una extensa cobertura para afianzar el carácter violento de la protesta. Por el contrario, los muertos y heridos de los manifestantes a manos de las fuerzas de seguridad del Estado fueron, en el mejor de los casos, reflejados como una simple cifra.

Muestra de ello fue el editorial publicado por El Comercio el 11 de octubre que reflejó a la víctima digna de la siguiente manera: “Una cobarde agresión sufrió el periodista de Teleamazonas, Freddy Paredes. Él cumplía su trabajo de reportería y decidió salir del lugar cuando fue atacado por detrás con una piedra que le causó heridas. Un enérgico rechazo y solidaridad".

Mientras que las víctimas indignas recibieron otro tipo de cobertura: "El país amaneció con la denuncia de la muerte de dos indígenas. No se han esclarecido las causas y en la tarde hubo el sepelio de uno de ellos" (El Comercio, "El país requiere de diálogo y mediación", 2019).

Otra víctima digna de las protestas fueron las fuerzas de seguridad del Estado que son retratadas como símbolo del resguardo de la paz y la seguridad, en contraposición con los indígenas y sus reivindicaciones que en ocasiones son tratados con un desdén que podría juzgarse de racista, como ocurre en columnas de opinión de El Universo publicada el 11 de octubre en las que se relató el desarrollo de las manifestaciones de la siguiente manera: "Felizmente, el Ejército y la Policía están actuando con prudencia y el principio de la reacción según la acción. Lo que vemos en la televisión es un buen control de la fuerza pública, que espero se mantenga para no dar el pretexto del "muertito" que exacerbe los ánimos" (Peña, 2019).

Por último, mencionar la víctima indigna del correísmo que es retratada en múltiples columnas de opinión como el responsable de la violencia, como se puede apreciar en columnas de opinión como la publicada por el diario El Comercio el 13 de octubre:

Rafael Correa no lanzó la piedra que hirió a Freddy ni las miles de piedras que se han lanzado contra nuestros policías y militares. Pero él, como Padrino del Odio, creó el ambiente para que este país se rompa en dos, "nosotros" los buenos y "ellos" los que odiamos. Y no lancemos más piedras, eso sería hacerle el juego al Gran Odiador. (Albornoz, 2019). 


\section{Conclusiones}

Tras realizar la presente investigación consideramos la plena vigencia del MP creado por Chomsky y Herman hace más de 30 años, aunque haya sufrido adaptaciones con paso del tiempo.

Se cumplen las hipótesis planteadas, tanto la conformidad de los medios de comunicación con los intereses de las élites económicas y políticas, como la aplicación de Ios distintos filtros establecidos en el MP. Finalmente, también hemos podido verificar cómo a raíz de los mencionados intereses y de conformidad con el intento de generar consensos en las audiencias, los medios construyen víctimas dignas e indignas.

\section{Bibliografía}

Admetricks (17 de Agosto de 2018). Admetricks. Obtenido de Admetricks: https:// blog.admetricks.com/ecuador-inversion-publicitaria-online-industriatelecomunicaciones-julio-2018/

Albornoz, V. (13 de Octubre de 2019). El Padre del Odio. Quito, Pichincha, Ecuador.

Alford, M. (2009). A Propaganda Model for Hollywood. Westminster Papers in Communication and Culture, 6(2), 144-156.

Álvarez-Peralta, M. (2018). CHAPTER 8 From \#15M to Podemos: Updating the Propaganda Model for Explaining Political Change in Spain and the Role of Digital Media. En J. Pedro-Carañana, D. Broudy, \& J. Klaehn, The Propaganda Model Today (págs. 107-124). London: University of Westminster Press.

Bagdikian, B. (1983). The Media Monopoly. Boston: Beacon Press.

Bergman, T. (2014). The Case for a Dutch Propaganda Model. International Journal of Communication, 8, 2705-2724.

Boyd-Barrett, 0. (2004). Judith Miller, The New York Times, and the Propaganda Model. Journalism Studies, 5(4), 435-449.

Broudy, D., \& Tanji, M. (2018). System Security: A Missing Filter for the Propaganda Model? En J. Pedro-Carañana, D. Broudy, \& J. Klaehn, The Propaganda Model Today (págs. 93-106). London: University of Westminster Press. 
Casado, F., \& Sánchez, R. (2017). La comunicación en tiempos de la Revolución Ciudadana: hablan los periodistas. Quito: CIESPAL.

Chomsky, N., \& Herman, E. (1988). Los guardianes de la libertad. Barcelona: Crítica.

Chomsky, N., Majfud, J., Castells, M., \& Cafassi, E. (3 de Octubre de 2019). Noam Chomsky sobre la crisis del neoliberalismo en Ecuador. Buenos Aires, Buenos Aires, Argentina.

Comercio, E. (11 de Octubre de 2019). El país requiere de diálogo y mediación. Quito, Pichincha, Ecuador.

---- (31 de Octubre de 2019). Lenín Moreno pide estrategia regional para defender la democracia. Quito, Pichincha, Ecuador.

--- (3 de Octubre de 2019). Youtube. Obtenido de Youtube: https://www. youtube.com/watch?v=VQSYbhnl8Go

Corner, J. (2003). Debate: The Model in Question. A Response to Klaehn on Herman and Chomsky. European Journal of Communication, 18(3), 367-375.

Ecuador, D. d. (16 de Octubre de 2019). Defensoría del Pueblo de Ecuador. Obtenido de Defensoría del Pueblo de Ecuador: https://www.dpe.gob.ec/ comision-de-justicia-y-reparacion-integral-de-la-defensoria-del-pueblo-sera-laencargada-de-transparentar-vulneraciones-de-los-derechos-humanos-durante-elparo-nacional-de-octubre-de-2019/

EFE (14 de Octubre de 2019). Asambleístas Soledad Buendía y Carlos Viteri Gualinga y otras 4 personas están en la Embajada de Ecuador en México. Quito, Pichincha, Ecuador.

Freedman, D. (2009). 'Smooth Operator?' The Propaganda Model and Moments of Crisis. Westminster Papers in Communication and Culture, 6(2), 59-72.

Good, J. E. (2008). The Framing of Climate Change in Canadian, American, and International Newspapers: A Media Propaganda Model Analysis. Canadian Journal of Communication, 33(2), 233-255. 
Herman, E. (2009). The Propaganda Model: a retrospective. Journalism Studies, $1(1), 101-112$.

Insider (27 de marzo de 2019). Zenith: la inversión en publicidad durante el 2019 representará el $49 \%$ del total global.

Kennis, A. (Diciembre de 2009). Synthesizing the Indexing and Propaganda Models: An Evaluation of US News Coverage of the Uprising in Ecuador, January 2000. Communication and Critical/Cultural Studies, 6(4), 386-409.

Klaehn, J. (2003). Behind the Invisible Curtain of Scholarly. Criticism: revisiting the propaganda model. Journalism Studies, 4(3), 359-369.

(2003). Debate: Model Construction: Various Other Epistemological Concerns. A Reply to John Corner's Commentary on the Propaganda Model. European Journal of Communication, 18(3), 377-383.

--- (2009). The Propaganda Model: Theoretical and Methodological Considerations. Westminster Papers in Communication and Culture, 6(2), 43-58.

(2018). Media, Power and the Origins of the Propaganda Model: An Interview with Edward S. Herman. Media Theory, 2(2), 95-100.

Labio-Bernal, A. (2018). Anti-Communism and the Mainstream Online Press in Spain: Criticism of Podemos as a Strategy of a Two-Party System in Crisis. En J. Pedro-Carañana, D. Broudy, \& K. Jeffery, The Propaganda Model Today (págs. 125141). London: University of Westminster Press.

Lang, K., \& Engel Lang, G. (2004). Response to Herman and Chomsky. Political Communication, 109-111.

Lester, E. (1992). Manufactured Silence and the Politics of Media Research: A Consideration of the "Propaganda Model". Journal of Communication Inquiry, 16 (1), 45-55.

Lukin, A. (2013). Journalism, ideology and linguistics: The paradox of Chomsky's linguistic legacy and his 'propaganda model'. Journalism, 14 (1), 96-110. 
Mullen, A. (2018). The 2008 Financial Crisis, the Great Recession and Austerity in Britain: Analysing Media Coverage Using the Herman-Chomsky Propaganda Model. En J. Pedro-Carañana, D. Broudy, \& J. Klaehn, The Propaganda Model Today (págs. 193-221). London: University of Westminster Press.

Orozco, M. (21 de Octubre de 2019). Celulares, tabletas y computadoras se comenzaron a importar con $0 \%$ de arancel. Quito, Pichincha, Ecuador.

Pedro, J. (2009). Evaluación crítica del modelo de propaganda de Herman y Chomsky. Revista Latina de Comunicación (64), 210-227.

Peña, E. (11 de Octubre de 2019). ¿Quién responde? Quito, Pichincha, Ecuador.

Pineda, A. (2001). El modelo de propaganda de Noam Chomsky: medios mainstream y control del pensamiento. Ámbitos, 191-210.

Pollick, B. (2018). The Sport of Shafting Fans and Taxpayers: An Application of the Propaganda Model to the Coverage of Professional Athletes and Team Owners. En J. Pedro-Carañana, D. Broudy, \& J. Klaehn, The Propaganda Model Today (págs. 173190). London: University of Westminster Press.

Pueblo, D. d. (9 de Octubre de 2019). Defensoría del Pueblo. Obtenido de Defensoría del Pueblo: https://www.dpe.gob.ec/la-defensoria-del-pueblo-entrega-a-la-corteconstitucional-informe-preliminar-de-control-del-estado-de-excepcion/

Rai, M. (2018). Thinking the Unthinkable about the Unthinkable - The Use of Nuclear Weapons and the Propaganda Model. En J. Pedro-Carañana, D. Boudry, \& J. Klaehn, The Propaganda Model Today (págs. 263-277). London: University of Westminster Press.

Reig, R. (2011). Los dueños del periodismo. Claves de la estructura mediática mundial y de España. Barcelona: Gedisa.

Robinson, P. (2018). Does the Propaganda Model Actually Theorise Propaganda? En J. Pedro-Carañana, D. Broudy, \& J. Klaehn, The Propaganda Model Today (págs. 53-67). London: Universitiy of Westminster.

Serrano, P. (2010). Traficantes de información. La historia oculta de los grupos de comunicación españoles. Madrid: Akal. 
Sierra, F. (2018). Imperialism and Hegemonic Information in Latin America: The Media Coup in Venezuela vs. the Criminalization of Protest in Mexico. En J. Pedro-Carañana, D. Boudry, \& J. Klaehn, The Propaganda Model Today (págs. 237-247). London: University of Westminster Press.

Sparks, C. (2007). Extending and Refining the Propaganda Model. Westminster Papers in Communication and Culture, 4(2), 68-84.

Sputnik (5 de Octubre de 2017). Presidente de Ecuador anuncia que realizará más consultas populares. Quito, Pichincha, Ecuador.

(30 de Octubre de 2019). Moreno: FFAA de Ecuador perdieron más de 9 millones de dólares en las protestas. Quito, Pichincha, Ecuador.

Taylor Jackson, P., \& Ronald Stanfield, J. (2004). The Role of the Press in a Democracy: Heterodox Economics and the Propaganda Model. Journal of Economic Issues, 38(2), 475-482.

Thompson, P. (2009). Market Manipulation? Applying the Propaganda Model to Financial Media Reporting. Westminster Papers in Communication and Culture, 6(2), 73-96.

Tv, E. (7 de Octubre de 2019). Youtube. Obtenido de Youtube: https://www.youtube.com/ watch?v=u9T4vrym9Ac

Universo, E. (9 de Octubre de 2019). Estado de excepción, sin decreto derogatorio. Guayaquil, Guayas, Ecuador.

---- (2019 de Octubre de 2019). Fiscalía allana instalaciones de Radio de la Prefectura de Pichincha por incitación a la discordia entre ciudadanos. Guayaquil, Guayas, Ecuador.

---- (14 de Octubre de 2019). Paola Pabón, Prefecta de Pichincha, fue detenida para investigaciones. Guayaquil, Guayas, Ecuador. 